\title{
Research Progress in the Clinical Pathogenesis and Treatment of Acute Kidney Injury
}

\author{
Yingying Liu, Qi Jiang* \\ Department of Nephrology, China-Japan Union Hospital of Jilin University, Changchun, Jilin, 130033, \\ China \\ *corresponding author
}

Keywords: acute kidney injury; clinical onset of a disease; research progress in treatment; clinical value

\begin{abstract}
Acute kidney injury is a common clinical syndrome and a serious complication of many diseases. It is a kind of critical illness, which seriously endangers patients' life safety and has a high mortality rate. This paper summarized the clinical treatment of acute kidney injury and reviewed the prognosis of acute kidney injury with studying the clinical pathogenesis of acute kidney injury to provide reference for the clinical diagnosis and treatment of acute kidney injury.
\end{abstract}

\section{Introduction}

The basic physiological function of the kidney is to eliminate the waste after metabolism. Therefore, the rapid deterioration of kidney function in a short period of time can have a serious impact on human health. If the condition is critical, it may even endanger the patient's life safety. According to medical investigations, the number of patients with acute kidney injury has increased year by year in recent years. In the United States, the recent incidence of acute kidney injury is at least three times higher than that of 20 years ago, and the number of patients with severe acute kidney injury accounts for about $50 \%$ of the total number of critically ill patients. This has contributed to a significant increase in medical costs. At present, acute kidney injury has become a prominent public health problem in the world. China has become the hardest-hit area for acute kidney injury. Acute kidney injury occurs mostly in patients with sepsis, heart surgery and other related diseases. It refers to a sudden and sustained decline in renal function, and serum creatinine rises $\geq 0.5 \mathrm{mg} / \mathrm{dl}$, which is represented by water electrolytes disorder, acid-base disturbance, azotemia and systemic dysfunction. Some patients may have oliguria or anuria. Acute kidney injury has a higher mortality rate and a poor prognosis. Strengthening early targeted treatment is an important way to improve the prognosis of patients. To further assist in the clinical diagnosis and treatment of patients with acute kidney injury, the following is the review of the pathogenesis, clinical diagnosis grading, treatment progress and prognosis of acute kidney injury.

\section{Pathogenesis of Acute Kidney Injury}

Acute kidney injury (AKI) is a group of clinical syndromes that act on different parts of kidney tissue according to the etiology and can be divided into pre-renal, post-renal, and renal categories. Pre-renal AKI is mainly associated with inadequate kidney perfusion caused by insufficient blood volume, decreased cardiac output, severe renal vasoconstriction, and renal arterial atresia. Post-renal AKI is caused by urethral obstruction caused by any level of the renal pelvis to the urethra. Renal AKI usually refers to renal parenchyma or renal vascular disease-associated AKI. Clinically, the pathogenesis of AKI varies.

The first is ischemia and reperfusion injury: ischemia and reperfusion injury is important pathogenesis of clinical AKI, which is common in organ transplantation, toxic shock, disseminated intravascular coagulation and acute blood loss. Especially in organ transplantation, it is almost inevitable and is the main cause of early functional recovery and transplant rejection. The kidney is a high perfusion organ. After renal ischemia, a large amount of ROS is produced during reperfusion, 
and a series of cellular reactions are triggered, which eventually leads to renal ischemia and reperfusion injury. Clinical and animal model studies have shown that IRI is inextricably linked to a large number of reactive oxygen species production, intracellular Ca 2+ overload, inflammatory response and apoptosis.

The second is the production of a large amount of ROS, which are oxygen molecules with high biological activity, such as oxygen ions, oxygenated free radicals, peroxides (such as hydrogen peroxide). According to different sources, they are divided into exogenous and endogenous sources. Exogenous ROS are derived from exogenous oxidants (ketones, nitros, etc.) or exogenous stimuli (drug oxidation, light, ionization, thermal radiation shock, etc.); endogenous ROS are derived from aerobic metabolism (xanthine oxidase metabolism, active neutrophile granulocyte, etc.) in living cells. When renal tissue is reperfused after ischemia, a large amount of ROS is produced in the body, which is one of the main causes of IRI. The specific mechanism is as follows: ROS damages biofilms, resulting in decreased fluidity and increased permeability. This leads to biofilm functional disorders (such as cell membrane rupture, mitochondrial membrane swelling and lysis, lysosomal membrane lysis, etc.); ROS increase causes nucleic acid damage. Possibly as ROS attacks nucleic acid bases or glycosidic bonds, DNA chains break, causing impaired cell function; ROS induces apoptosis, leading to intracellular Ca 2+ migration and increasing intracellular cytosolic Ca 2+ concentration. This exacerbated the occurrence of AKI.

The third is intracellular Ca 2+ overload. Intracellular Ca 2+ overload is an important factor in AKI caused by ischemia or reperfusion. ROS promotes internal flow of $\mathrm{Ca} 2+$, increasing intracellular $\mathrm{Ca} 2+$ concentration; Ca 2+ activates proteolytic enzymes and phospholipase A 2, promoting the ROS disruption. The two synergistically interact with each other, leading to an increase in renal tissue damage. With ischemia in the kidney tissue, $\mathrm{Na}+, \mathrm{K}+$-ATPase activity decreases during reperfusion, promoting the increase of $\mathrm{H}+$, internal flow of $\mathrm{Na}+$ influx, activation of $\mathrm{Na}+/ \mathrm{Ca} 2+$ exchange system. This aggravates intracellular $\mathrm{Ca} 2+$ accumulation, resulting in uneven intracellular ion distribution, affecting regulatory functions, enhancing mitochondrial permeability, decreasing membrane potential, which will impair function of the organ. This results in inhibition of ATP synthesis, energy synthesis defect, triggering cascade damage response. In addition, the increase of $\mathrm{Ca} 2+$ concentration in cytoplasm will activate $\mathrm{Ca} 2+/ \mathrm{Mg} 2+$-dependent endonuclease, break DNA chain , impair cell function and induce apoptosis. It can be seen that during renal ischemia and reperfusion, intracellular Ca $2+$ overload and the production of large amounts of ROS reinforce each other, which becomes a key influencing factor for acute kidney injury.

The fourth is the inflammatory response. The inflammatory cascade response is another major cause of acute renal tissue decline after ischemia and reperfusion. After ischemia and reperfusion, inflammatory cells and renal endothelial cells can be activated to increase expressions of cytokines (interleukins, chemotactic cytokines, tumor necrosis factors, etc.) and adhesive factors (including integrin family, cadherin family and immune globulin family). TNF- $\alpha$ is a key factor triggering the initiation of many cytokines and even systemic inflammatory response. It can directly induce the release of inflammatory cytokines such as interleukin-1, interleukin-6 and interleukin-8, triggering the inflammatory cascade response. At the same time, it also promotes the expression of adhesive factors and enhances the adhesion between inflammatory cells and endothelial cells, neutrophil infiltration and leukocyte activation to cause inflammation. Mast cells release a large number of inflammatory mediators (eosinophilic granulocyte chemotactic factors, prostaglandin D2, leukotrienes and some cytokines, etc.). Then the cascade reaction amplified the signal step by step, eventually causing cell physiological dysfunction and inflammation spread.

\section{Clinical Diagnosis Grading of Acute Kidney Injury}

There is no unified definition of acute kidney injury clinically and the acute kidney injury in the past is called acute renal failure. There exist many clinical definitions of acute renal failure, which directly leads to the difficulty in clinical diagnosis of acute kidney injury. This not only affects judgment of the patient's condition, but also affects the patient's treatment process. In addition, the 
causes and mechanisms of acute kidney injury are very complicated. Many common causes interact with each other, thus causing acute kidney injury. For example, in the study of pathophysiology, renal and pre-renal types of acute kidney injury may belong to successive pathological changes or the same disease spectrum, which may be causal or exist in the same time period. Currently, the division of the two types in clinic is not clear. However, it cannot be ignored that there are many clinical classifications of renal failure, which can be divided into different sub-types. Generally, at least 30 types of acute kidney injury and disease progression evaluation criteria are used. The cause of acute kidney injury was originally thought to be related only to oliguria. That is to say when the urine volume was less than $400 \mathrm{ml}$ in 24 hours, acute kidney injury could be judged. But medical research and many years of practice confirmed that the symptoms of oliguria may occur after least tens of hours or weeks with the onset of acute kidney injury, which means oliguria is not an early symptom of acute kidney injury, and has no significance for the early diagnosis of acute kidney injury. In the past ten years, due to the continuous improvement of medical level, more than half of patients with acute kidney injury have no oliguria symptoms. Therefore, the way to diagnose cute kidney injury by oliguria is gradually eliminated. Since the physiological function of the human kidney is to excrete physiological metabolic waste, the filtration function of the basic tissue unit of the kidney is considered by many scholars to be the most diagnostic indicator reflecting the function of the kidney. Therefore, the examination of exogenous markers is often carried out to assess human kidney function, such as inulin, 131I-labeled brain shadow muriatic acid, and iohexol. Although the accuracy is high, the inspection steps are complicated. Special instruments and drugs are needed, so it is more difficult to promote it and only large and medium-sized medical institutions can use it.

\section{Treatment of Acute Kidney Injury}

In the past, acute kidney injury was often treated by controlling risk factors of acute kidney injury, such as controlling infection, improving blood volume, improving body balance, and preventing shock for patients. At present, the clinical understanding of acute kidney injury is continuously strengthened. In addition to symptomatic treatment for acute kidney injury, renal replacement therapy is mainly implemented. Kidney replacement therapy mainly includes kidney transplantation and blood purification. The cost of kidney transplantation is high with higher technical requirements, so there is few clinical application; blood purification treatment is relatively simple and the clinical application is more extensive. Current blood purification methods include intermittent renal replacement therapy (IRRT), peritoneal dialysis (PD), continuous renal replacement therapy (CRRT), and heterozygous renal replacement therapy (HRRT).

IRRT is a traditional treatment for acute kidney injury. The hemodynamics of the patient is stable during treatment. The effect on solute control is significant, and the effect of clearing the liquid is obvious. However, how to make the hemodynamic index to remain stable is the largest problem in this treatment method and nearly $30 \%$ of patients have this problem.

PD mainly uses the human body's own peritoneum as a dialysis membrane for blood purification. This method can effectively reduce the risk of anticoagulation bleeding compared with traditional hemodialysis. However, this method is less effective for small molecules and water scavenging, and for patients with pulmonary edema and hyperkalemia. In addition, long-term implementation of PD treatment is likely to lead to peritonitis.

CRRT is a kidney replacement treatment program that emerged in the 1980s. It has a significant effect on the clearing of water retention, inflammatory factors and metabolic waste. It can effectively maintain the balance and stability of the body and is suitable for patients with instability of hemodynamic parameters. But CRRT use more heparin than IRRT, which is more likely to affect hemodynamic stability and has a higher risk of bleeding. It is still uncertain that CRRT can significantly improve the survival rate of patients with acute kidney injury compared with IRRT. However, CRRT can achieve good clinical results for patients with sepsis, high catabolism, high intracranial pressure, cerebral edema, and excessive volume load.

HRRT is a new treatment based on IRRT and CRRT. It combines the therapeutic advantages of IRRT and CRRT to improve the elimination of body waste and reduce the continuous heparin dose 
to reduce the risk of bleeding.

\section{References}

[1]Yin Lianhong, QI Meng, Peng Jinyong. Research Progress on Molecular Mechanism and Intervention of Traditional Chinese Medicine of Acute Kidney Injury [J]. Chinese Pharmacological Bulletin, 2016, 32(11): 1494-1500.

[2] Yao Yuan. Progress in the Treatment of Acute Kidney Injury with Sepsis by Continuous Renal Replacement [J]. Journal of Clinical Medical Literate, 2017, 4(38): 7523-7524.

[3] Di Haoran, Lu Youran, Wu Yanqing, Zhang Lei, Shao Fei, Lu Xiaoqin, Guo Yuhong and Liu Qingquan. Pathogenesis and TCM Treatment of Acute Kidney Injury with Sepsis [J]. World Chinese Medicine, 2018, 13(08): 2079 -2083+2089.

[4] Luo Gang, Yang Xu. Pathogenesis and Treatment Progress of Renal injury Associated with Severe Acute Pancreatitis [J]. Chinese Community Doctors, 2015, 31(08): 8-9+11. 